Article

\title{
Smartphone-guided educational counseling and self-help for chronic tinnitus
}

\author{
Winfried Schlee ${ }^{1, *}$, Patrick Neff ${ }^{1,2}$, Jorge Simoes ${ }^{1}$, Berthold Langguth ${ }^{1}$, Stefan Schoisswohl ${ }^{1}$, Heidi Steinberger ${ }^{1}$, \\ Marie Norman', Myra Spiliopoulou ${ }^{3}$, Johannes Schobel ${ }^{4}$, Ronny Hannemann ${ }^{5}$ and Rüdiger Pryss ${ }^{6}$
}

\author{
1 Department of Psychiatry and Psychotherapy, University of Regensburg, 93053 Regensburg, Germany \\ 2 URPP "Dynamics of Healthy Ageing", University of Zürich, 8006 Zürich, Switzerland \\ 3 Knowledge Management and Discovery Lab, Otto-von-Guericke University, 39106 Magdeburg, Germany \\ 4 DigiHealth Institute, Neu-Ulm University of Applied Sciences, 89231 Neu-Ulm, Germany \\ 5 WSAudiology, Sivantos GmbH, 91058 Erlangen, Germany \\ 6 Institute of Clinical Epidemiology and Biometry, University of Würzburg, 97080 Würzburg, Germany \\ * Correspondence: winfried.schlee@gmail.com
}

\begin{abstract}
:
Tinnitus is an auditory phantom perception in the ears or head in the absence of a corresponding external stimulus. There is currently no effective treatment available that reliably reduces tinnitus. Educative counseling is a treatment approach that aims to educate patients and inform them about possible coping strategies.

For this feasibility study, we implemented educational material and self-help advice in a smartphone app. Participants used the educational smartphone unsupervised during their daily routine over a period of 4 months.

Comparing the tinnitus outcome measures before and after smartphone-guided treatment, we measured changes in the tinnitus-related distress, but not in tinnitus loudness.

Improvements on the Tinnitus Severity numeric rating scale reached an effect size of .408, while the improvements on the THI were much smaller with an effect size of .168.

Analysis on the user behavior showed that frequent and intensive use of the app is a crucial factor for treatment success: participants that used the app more often and interacted with the app intensively, reported a stronger improvement of the tinnitus.

Between study allocation and final assessment, 26 of 52 participants dropped out of the study. Reasons for the dropouts and lessons for future studies are discussed in this paper.
\end{abstract}

Keywords: tinnitus; self-help; ecological momentary assessment; ehealth; smart-phone; intervention

\section{Introduction}

Tinnitus is an auditory phantom perception in the ears or head in the absence of a corresponding external stimulus $[1,2]$. With a prevalence of $8-28 \%$, the condition is very common in western societies $[3,4]$, and $1-4 \%$ of the society reports that they are severely suffering from tinnitus $[3,5,6]$. In those severe cases, tinnnitus is frequently associated with depression, anxiety, insomnia, concentration difficulties and poor psychological health, all of which have a significant impact on their quality of life [6,7]. The term "tinnitus disorder" has recently been suggested to describe these severe cases where auditory phantom perception is associated with subjective suffering of the affected people [2]. 
Currently, there is no effective treatment available that reliably reduces auditory perception of tinnitus. Reviewing the progress of clinical research in the field of tinnitus, several authors [8-11] highlighted the large patient heterogeneity as one of the leading reasons for inconsistent results in clinical research hampering the development of a uniformly effective treatment againt tinnitus. In a review by Baguley et al., at least 13 different types of causal factors for tinnitus have been identified [6]. It is supposed that these different etiologic factors result in various subtypes of tinnitus with differences in their pathophysiology, which in turn may require different treatment strategies. Even though effective treatments for reducing the sound perception need further research and development - effective treatments for reducing the subjective suffering of the tinnitus patients already exist. In a recent Cochrane review, Fuller and colleagues [12] analyzed the effectiveness of Cognitive Behavioral Treatment (CBT) and reported an average effect size of .56 for reducing the impact of tinnitus on quality of life at end of treatment.

Educative counseling is a treatment approach that aims to educate chronic tinnitus patients and inform them about possible coping strategies. By means of education, counseling, and informing the patient about tinnitus, the patient should be helped to better understand the underlying causes of the conscious tinnitus perception and to deal with possible accompanying symptoms like sleep disturbances, concentration problems, etc. Such an educational counseling approach aims to promote self-help capacities of the patients and enhance patient empowerment. In principle, educational counseling can be applied as a solitary therapy or in combination with other clinical interventions [13].

In a multicenter randomized clinical trial, Henry and colleagues [14] enrolled 148 veterans with chronic tinnitus and randomized them to a tinnitus masking treatment $(\mathrm{n}=$ $42)$, tinnitus retraining therapy $(n=34)$, tinnitus educational counseling $(n=39)$ or a waiting list control $(n=33)$. Tinnitus severity was significantly reduced in the tinnitus masking, tinnitus retraining therapy and tinnitus educational counseling group compared to the waiting list group directly after end of treatment as well as after a 6 month follow up period. Comparison between the treatment groups did not reveal a difference of statistical significance, showing that educational counseling alone is similarly effective like tinnitus masking and tinnitus retraining treatment. In a systematic review, Xiang et al. [15] analyzed nine clinical studies (the Henry study included) to compare stand-alone educational counseling with other psychological interventions or combination therapies and did not find a significant difference between the interventions. Even though the clinical practice guideline (CPG) of the American Academy of Otolaryngology-Head\&Neck Surgery foundation [16] as well as the European Tinnitus Guidelines [17] recommend educational counseling as one of the treatment options for chronic tinnitus, systematic research on educational counseling in tinnitus is still limited.

There are several major challenges with studies on educational counseling. The first challenge is the choice of a control condition, the second challenge is the standardization of the intervention across participants and the third challenge the assessment of the active cognitive involvement of the individual participants in the educational process. Improvements of tinnitus severity following educational counseling and and the implementation of self-help in the daily life are most likely a gradual process that needs to be monitored over a longer period.

In this study, our strategy was to implement educational material and self-help advice in a smartphone app and measure tinnitus symptoms daily for a period of 4 months. Every day, the participants receive a new advice for tinnitus self-management together with an explanation of the medical background for it. With this study we aimed to investigate the clinical improvement induced by an electronic tool for systematic educational counseling and self-help in tinnitus and to research the relationship between active user involvement and clinical improvement. 


\section{Materials and Methods}

\subsection{Study Design}

For this fesability study, we report two samples that have used the same app-based educational counseling with with similar study design outcome assessment (figure 1). In the first run, participants were randomized to treatment arms 1 and 2 . In treatment arm 1 , the patients received four months of smartphone-guided eductional counceling treatment plus Ecological Momentary Assessment (EMA). In treatment arm 2, patients received for two months EMA only, followed by a period of another two months where the patients received the smartphone-guided eductional counceling treatment plus EMA. In the second run (treatment arm 3), there was no randomization and all patients received four months of smartphone-guided eductional counceling treatment plus Ecological Momentary Assessment (EMA). The study protocol was reviewed by the Research Ethics Committees of the University Hospital Regensburg (protocol number: 17-544_1-101). The app was implemented for iOS devices. The aim was to test the feasibility of app-based educational counseling treatment on a clinical tinnitus population where the participants use the smartphone unsupervised during their daily routine.

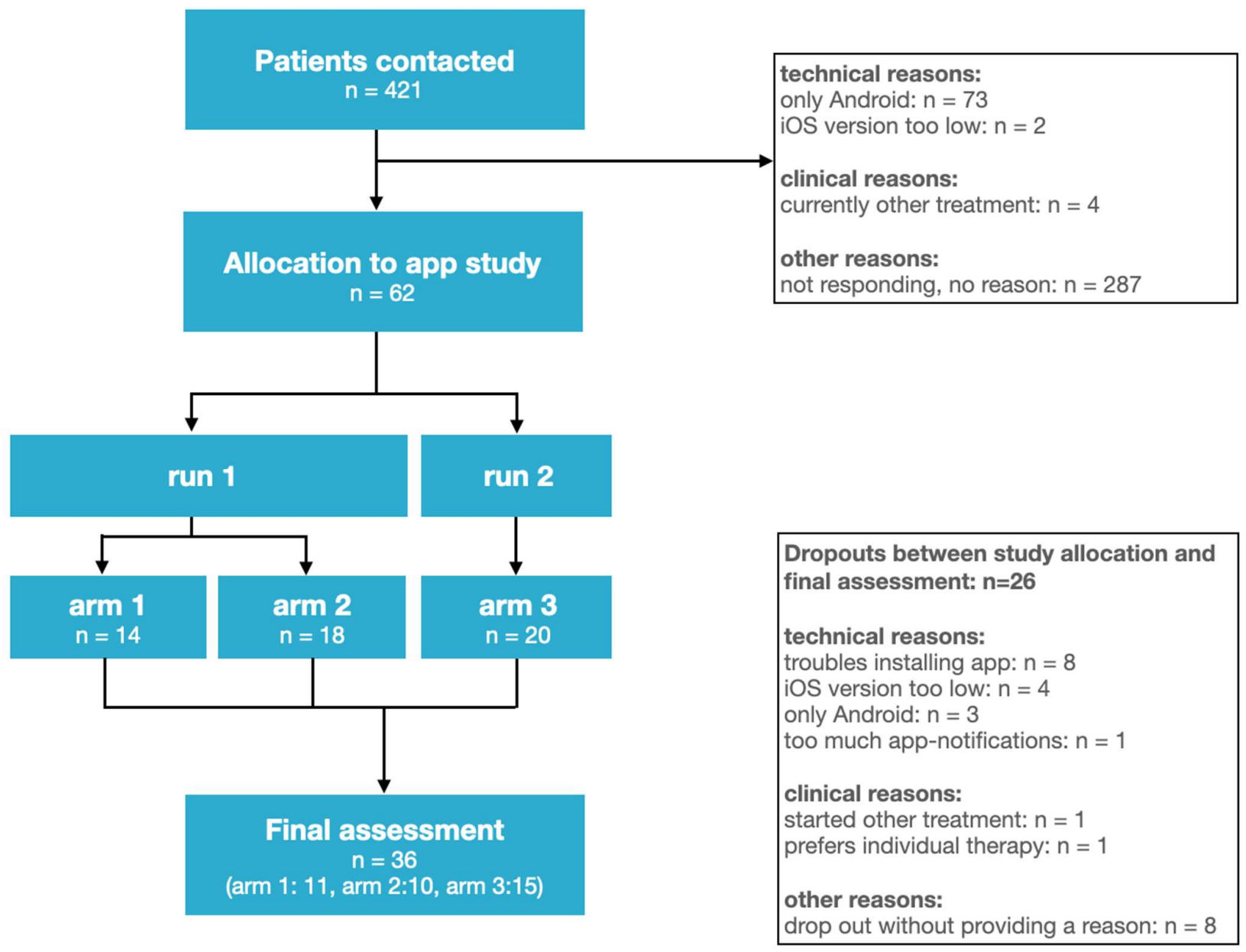


Figure 1. Participant flow diagram. In treatment arm 1 and 3, patients received four months of smartphone-guided educational counceling treatment plus Ecological Momentary Assessment. In treatment arm 2, patients received for two months, Ecological Momentary Assessment only, followed by a period of another two months where the patients received the smartphone-guided educational counceling treatment plus Ecological Momentary Assessment.

\subsection{Smartphone-guided educational counseling}

During the treatment phase, participants received every day a "tip of the day". The tips were randomly assigned from a library of 108 self-help tipps and the tip was displayed after completion of the EMA questionnaire.

The tips were designed to be concise and succinct so that they would preferably not exceed the display size of an average smartphone and that reading the tips would be possible without spending a lot of time in everyday life. Furthermore, the tips should also be as detailed and informative as possible in order to convey the content to the patients in an understandable way. For this reason, the tips were structured in a uniform manner: Each self-help tip consists of a title, an objective, the tip itself, and a more detailed explanation of the tip. The "title" helps the respondent find a tip again if needed and gives a brief insight into the content of the tip. The "objective" summarizes the tip in one sentence. The section with the actual tip contains the essential information, recommendations, and hints. In the explanation section, the reader can find background knowledge or supplementary information about the tip. This structure allows the respondent to quickly decide which sections of the tip are relevant and worth reading, depending on the available time and interest. The tipps were on average 101 words long (range 40 to 155 words). The tips covered topics like e.g. the influence of sport on tinnitus, self-help groups, the use of noisers, cognitive appraisal, influence of emotions and how to modulate, music listening, relaxation of jaw and neck or noise protection. The tips were developed based on existing self-help guides for tinnitus [18-22] and underwent an internal review process before being implemented in the app.

The smatphone app was used in the testflight mode of iOS, which is a testing environment for smartphone apps. The apps in the testflight do not appear in the offical app store and have to be installed in a different way. To install the app, participants received an email with a link to install the "TestFlight" app und iOS. The "TestFlight" app in turn enabled the installation of the TinnitusTipps app. When opening the TinnitusTipps app for the first time, the user had to log in with their account credentials. Then they received the invitation to the respective study group, which had to be accepted. As support, study participants were sent an email explaining the installation process both in written form and in the form of a short video.

\subsection{Ecological Momentary Assessment}

Ecological Momentary Assessment (EMA) of the tinnitus was done using two questions on tinnitus loudness and tinnitus distress, using the questionnaire published by Schlee et al. 2016 [23]. In addition, patients were asked about their momentary hearing ability, their limitation due to hearing loss, and if they were using a hearing aid at the moment. Furthermore, they have been asked about the momentary perceived stress level, and exhaustion. The goal was to fill out the EMA questionnaire at three times during the day and the participants received random notifications to fill out the questions. The EMA data are reported in [24].

\subsection{Participants}

Participants were contacted by email or telephone and invited to participate in this study. Participants were selected from a list of chronic tinnitus patients from the tinnitus 
center Regensburg, which had previously indiciated their willingness to participate in studies on tinnitus. Only adult patients with chronic tinnitus of at least 6 months of duration were included in the study. The participants needed a smartphone running an iOS system greater than 10. Candidates were excluded in case of an acute psychosis, an acute major depression or a substance abuse disorder in the 12 weeks before starting treatment, epilepsy or other diseases of the central nervous system, or if they had undergone another treatment within three months before treatment start. Participants with pharmacological treatment were only included in case the medication was stable at least 10 days before treatment start.

\subsection{Measurement instruments}

The Tinnitus Handicap Inventory (THI) [25] and the Tinnitus Severity Numeric Rating Scale [8] are clinical outcome measures that are commonly used to assess tinnitus distress. The second question of the Tinnitus Severity Numeric Rating Scale is used to collect selfratings of the subjective tinnitus loudness. The THI questionaire served as the primary outcome measure for this study. All tinnitus questionnaires were assess before the beginning of the study and after the end of the 4-months-study.

The European School for Interdisciplinary Tinnitus Research Screening Questionnaire (ESIT-SQ) is an instrument to systematically assess the tinnitus medical history [26]. At the time of data collection, no validated tool was available to consistently measure a patient's level of empowerment. Thus, for the present study, the Tinnitus Empowerment Scale (TES) was designed based on patient empowerment questionnaires in diabetes [27] and psoriasis [28]. In this questionnaier, three questions asked about tinnitus literacy to assess how much the patient knows about tinnitus; three questions asked about coping skills for tinnitus management; three questions assessed how self-confident thinks and talks about tinnitus; three quesstions asked about the self-confidence in coping with the tinnitus; and three questions asked about the awareness of the cause for tinnitus and factors that influence the dynamic changes of the own tinnitus.

\subsection{Statistical analysis}

Statistical analyses were conducted in R [29], using R-version 4.0.3 (10 October 2020). Mixed models analysis were calculated using the nlme package (version 3.1-152). Handling of the data from the smarthpone app were done using the jsonlite packace (version 1.7.2). Missing values were coded as NA and removed for the respective analysis. The non-parametric Kruskal-Wallis test was used for analysing continuous variables whether the participants in the three arms originate from the same patient distribution. Chi-square tests were used to analyze count variables. Cohen's d effect size were calculated using the effsize package.

\section{Results}

\subsection{Characteristics and summary of study participants}

In total, 36 chronic tinnitus patients (14 female, 22 male) with an average age of 49.4 (SD 11.7) years finished the smartphone-guided educational treatment and were included in the final analysis. More details about the study groups at baseline are given in Table 1.

Table 1. Characteristics of participants in final analysis. $P$ values is based on Chi square test for count variables or Kruskal-Wallis test for continuous variables. 


\begin{tabular}{lcccccc}
\hline \multicolumn{1}{c}{ Characteristics } & Units & Full cohort & Arm 1 & Arm 2 & Arm 3 & $\boldsymbol{P}$ value \\
\hline $\begin{array}{l}\text { Participants per proto- } \\
\text { col }\end{array}$ & \# Participants & 36 & 11 & 10 & 15 & 0.433 \\
\hline Gender: female & \# Participants (\%) & $14(38.9)$ & $4(36.3)$ & $4(40.0)$ & $6(40.0)$ & 0.752 \\
\hline Gender: male & \# Participants (\%) & $22(61.1)$ & $7(63.6)$ & $6(60.0)$ & $9(60.0)$ & 0.728 \\
\hline Tinnitus duration & Years [mean (SD)] & $8.6(17.5)$ & $14.1(28.6)$ & $9.8(10.0)$ & $3.2(5.4)$ & 0.054 \\
\hline Age at baseline & Years [mean (SD)] & $49.4(11.7)$ & $49.6(9.8)$ & $51.7(12.7)$ & $47.6(12.6)$ & 0.381 \\
\hline THI at baseline & Points [mean (SD)] & $48.1(23.6)$ & $50.7(22.2)$ & $44.4(24.7)$ & $48.6(25.4)$ & 0.826 \\
\hline $\begin{array}{l}\text { NRS tinnitus distress } \\
\text { at baseline }\end{array}$ & Points [mean (SD)] & $2.5(1.0)$ & $2.5(1.0)$ & $2.8(0.9)$ & $2.2(1.0)$ & 0.400 \\
$\begin{array}{l}\text { NRS tinnitus loudness } \\
\text { at baseline }\end{array}$ & Points [mean (SD)] & $6.3(2.2)$ & $6.0(2.1)$ & $7.0(2.6)$ & $6.2(2.1)$ & 0.349 \\
\hline
\end{tabular}

\subsection{Within-arm and between-arm analysis of tinnitus symptoms}

THI sum score. A two-way mixed model ANOVA was performed to analyze the effect of the time point (pre vs. post) and group allocation on the THI sum score. Analysis of the main effects revealed that group allocation did not have a statistically significant effect on the THI sum score $(F(2,33)=.270, \mathrm{p}=.765)$ while the influence of the time point was close to the threshold of statistical significance $(F(1,31)=3.94, p=.056)$. The analysis revealed that there was no statistically significant interaction between the time point and group allocation $(\mathrm{F}(2,31)=.403, \mathrm{p}=.672)$.

For post hoc analysis, a Wilcoxon Signed Rank Test was calculated and revealed statistical significance between the pre and post intervention phase $(\mathrm{W}=345, \mathrm{p}=.02)$. Cohen's $d$ effect size was calculated with .168 (confidence interval: -.31 to .64 ).

Tinnitus severity 1: problem of tinnitus. A two-way mixed model ANOVA was performed to analyze the effect of the time point (pre vs. post) and group allocation on the TS1 score. Analysis of the main effects revealed that group allocation did not have a statistically significant effect on TS1 score $(\mathrm{F}(2,33)=.862, \mathrm{p}=.432)$ while the influence of the time point was statistically significant $(\mathrm{F}(1,31)=14.0, \mathrm{p}=<.001)$. The analysis revealed that there was no statistically significant interaction between the time point and group allocation $(\mathrm{F}(2,31)=3.14, \mathrm{p}=.057)$.

For post hoc analysis, a Wilcoxon Signed Rank Test was calculated and revealed statistical significance between the pre and post intervention phase $(\mathrm{W}=119, \mathrm{p}=.003)$. Cohen's $\mathrm{d}$ effect size was calculated with .408 (confidence interval: -.07 to .89 ).

Tinnitus severity 2: tinnitus loudness. A two-way mixed model ANOVA was performed to analyze the effect of the time point (pre vs. post) and group allocation on the TS2 score. Analysis of the main effects revealed that the group allocation did not have a statistically significant effect on the TS2 score $(F(2,33)=.265, \mathrm{p}=.769)$ neither the time point $(\mathrm{F}(1,31)=.187, \mathrm{p}=.668)$. The analysis revealed that there was no statistically significant interaction between the time point and group allocation $(F(2,31)=.952, \mathrm{p}=$ .397). 
Table 2. Results of the mixed effects models for tinnitus outcome measures

\begin{tabular}{|c|c|c|c|c|c|c|}
\hline \multirow[t]{2}{*}{ Outcome } & \multirow[t]{2}{*}{ Fixed effects } & \multirow[b]{2}{*}{ numDF/denDF } & \multirow[b]{2}{*}{ Fvalue } & \multicolumn{3}{|c|}{ Random effects } \\
\hline & & & & Pvalue & & $S D$ \\
\hline THI & Time & $1 / 31$ & 3.94 & .056 & participants & 22.1 \\
\hline \multirow[t]{2}{*}{ sum score } & Group & $2 / 33$ & .270 & .765 & residuals & 7.41 \\
\hline & Time*Group & $2 / 31$ & .403 & .672 & & \\
\hline \multirow{3}{*}{$\begin{array}{l}\text { TS1: } \\
\text { tinnitus } \\
\text { problem }\end{array}$} & Time & $1 / 31$ & 14.0 & $<.001$ & participants & .858 \\
\hline & Group & $2 / 33$ & .862 & .432 & residuals & .397 \\
\hline & Time* Group & $2 / 31$ & 3.14 & .057 & & \\
\hline TS2: & Time & $1 / 31$ & .187 & .668 & participants & 1.85 \\
\hline tinnitus & Group & $2 / 33$ & .265 & .769 & residuals & 1.25 \\
\hline loudness & Time ${ }^{*}$ Group & $2 / 31$ & .952 & .397 & & \\
\hline
\end{tabular}

\subsection{Influence of user engagement on the treatment effect}

A multiple linear regression analysis was calcuated to predict the THI change based on the user interaction (i.e. how often did the participant rate a tip) and total app usage (i.e. how often did the participant fill out a questionnaire). The interaction of the factors 'user interaction' and ,total usage' was a significant predictor of the THI change (table 3 ). The total usage was measured by the number of EMA questionnaires filled out by the participant. Within the app, the user had the possiblity to rate the tips with one to five stars; this user interaction was measured by the number of tip ratings done by the participant.

Table 3. User engagement of enrolled participants

\begin{tabular}{|c|c|c|c|c|c|}
\hline Outcome & & & & & \\
\hline \multirow{4}{*}{$\begin{array}{l}\text { THI } \\
\text { sum score }\end{array}$} & & Estimate & $S E$ & tvalue & Pvalue \\
\hline & User interaction & -1.41 & 2.07 & -0.682 & 0.501 \\
\hline & Total usage & 1.87 & 2.03 & 0.918 & 0.366 \\
\hline & $\begin{array}{l}\text { User interaction* } \\
\text { Total usage }\end{array}$ & -4.63 & 1.57 & -2.96 & 0.006 \\
\hline
\end{tabular}

\subsection{Analysis of changes in patient empowerment}

Health literacy. A two-way mixed model ANOVA was performed to analyze the effect of the time point (pre vs. post) and group allocation on the health literacy subscore. Analysis of the main effects revealed that the group allocation did not have a statistically significant effect on the health literacy subscore $(F(2,33)=.455, p=.639)$ while the influence of the time point was statisticaly significant $(F(1,30)=10.1, p=.004)$. The analysis revealed that there was no statistically significant interaction between the time point and group allocation $(\mathrm{F}(2,30)=.350, \mathrm{p}=.708)$.

For post-hoc analysis, a Wilcoxon Signed Rank Test was calculated and revealed a statistical significance between the pre and post intervention phase $(\mathrm{W}=428, \mathrm{p}=.042)$. Cohen's d effect size was calculated with .566 (confidence interval: .076 to 1.06). 
Coping. A two-way mixed model ANOVA was performed to analyze the effect of the time point (pre vs. post) and group allocation on the coping subscore. Analysis of the main effects revealed that the group allocation did not have a statistically significant effect on the coping subscore $(\mathrm{F}(2,33)=.310, \mathrm{p}=.736)$ while the influence of the time point was statisticaly significant $(\mathrm{F}(1,30)=4.59, \mathrm{p}=.040)$. The analysis revealed that there was no statistically significant interaction between the time point and group allocation $(\mathrm{F}(2,30)=$ $.817, \mathrm{p}=.451)$.

For post hoc analysis, a Wilcoxon signed rank test was calculated and revealed statistical significance between the pre- and post-intervention phase $(W=492, p=.216)$. Cohen's d effect size was calculated with .285 (confidence interval: -.198 to .769).

Confidence, Self efficacy, Self awareness. Two-way mixed model ANOVA were performed to analyze the effect of the time point (pre vs. post) and group allocation on the subscores of confidence, self efficacy and self awareness. Analysis of main effects and interaction effects did not reveal any statistically significant influence of time point or group allocation on these subscores. The results are reported in table 4 .

Table 4. Results of the mixed effects models for patient empowerment outcome measures

\begin{tabular}{|c|c|c|c|c|c|c|}
\hline \multirow{5}{*}{$\begin{array}{l}\text { Subscores } \\
\text { Health Literacy }\end{array}$} & \multicolumn{3}{|l|}{ Fixed effects } & \multicolumn{3}{|c|}{ Random effects } \\
\hline & & numDF/denDF & Fvalue & Pvalue & & $S D$ \\
\hline & Time & $1 / 30$ & 10.1 & .004 & participants & 1.35 \\
\hline & Group & $2 / 33$ & .455 & .639 & residuals & 1.39 \\
\hline & Time ${ }^{*}$ Group & $2 / 30$ & .350 & .708 & & \\
\hline \multirow{3}{*}{ Coping } & Time & $1 / 30$ & 4.59 & .040 & participants & 2.47 \\
\hline & Group & $2 / 33$ & .310 & .736 & residuals & 1.22 \\
\hline & Time* Group & $2 / 30$ & 0.817 & .451 & & \\
\hline \multirow{3}{*}{ Confidence } & Time & $1 / 30$ & .041 & .840 & participants & 1.49 \\
\hline & Group & $2 / 33$ & 1.02 & .373 & residuals & 1.30 \\
\hline & Time ${ }^{*}$ Group & $2 / 30$ & .667 & .521 & & \\
\hline \multirow{3}{*}{ Self-Efficacy } & Time & $1 / 30$ & 1.62 & .213 & participants & 2.55 \\
\hline & Group & $2 / 33$ & 1.01 & .375 & residuals & 1.32 \\
\hline & Time*Group & $2 / 30$ & .416 & .663 & & \\
\hline \multirow{3}{*}{ Self-Awareness } & Time & $1 / 30$ & 2.95 & .096 & participants & 1.83 \\
\hline & Group & $2 / 33$ & 2.26 & .120 & residuals & 1.67 \\
\hline & Time*Group & $2 / 30$ & .744 & .484 & & \\
\hline
\end{tabular}

\section{Discussion}

The main objective of this feasibility study was to test a novel smartphone-guided educational treatment with self-help tips for chronic tinnitus patients that are using the app unsupervised during their daily routine. Participants used the smartphone app for a period of four months in their everyday life without guidance or reminders by the study personal. 
Therefore, the study design represents a naturalistic setting of users that download a treatment app from the appstore and use the health app without medical guidance.

Changes of tinnitus. Comparing the tinnitus outcome measures before and after smartphone-guided treatment, we measured changes in the tinnitus-related distress, but not in tinnitus loudness. Improvements on the Tinnitus Severity numeric rating scale reached an effect size of .408 , while the improvements on the THI were much smaller with an effect size of .168. The changes in the tinnitus loudness have been far from any meaningful change. This suggests that TinnitusTipps treatment helped participants improve their coping skills and reduce psychological suffering while the tinnitus sound itself is still present and remains unchanged.

Importantly, the main effect for group did not reach the significance level in neither analysis, suggesting that the treatment duration of 2 months versus 4 months, did not discriminate. Future studies will be needed to identify the best treatment duration for the intervention. It is possible, that the duration of 2 months is already enough to reach the maximum possible treatment effect.

Relationship between user behavior and tinnitus change. The individual results of the participants appeared with a large variability. While some participants reported a strong reduction of tinnitus suffering during the treatment period, other participants reported no change or even worsening. An additional analysis on the user behavior revealed that the amount of app usage (i.e. count how often the participant filled out the questionnaires) together with the user interaction (i.e. how often did the user rate the tips) can predict the tinnitus improvement. Interestingly, neither the main effect of total usage nor the main effect of user interaction explained the variance of the tinnitus changes significantly. The interaction effect of total usage and interaction, however, explained the changes in tinnitus distress significantly. This means that the frequent and intensive use of the app is a crucial factor for treatment success: participants that used the app more often and interacted with the app intensively, reported a stronger improvement of the tinnitus. We suggest that future studies track more details of the user behavior to allow additional analyses on the relationship between user behavior and tinnitus improvement. If intensive use of the health app was linked to better tinnitus improvement, one might hypothesize about a causal interaction and consequently try to increase the attractiveness of the app and motivate users to regular use of the app. After end of treatment, participants were asked with open-ended questions about how to improve the app. Individual users reported that they would prefer less EMA questions during the day and that some questions (e.g. question on hearing aid) did not fit to their individual situation. This user feedback suggests, that more individualized EMA sampling and less questionnaires could help to increase the app usage.

Changes in patient empowerment. Patient empowerment was measured with the custom questionnaire "Tinnitus Empowerment Scale (TES)". The subscales 'health literacy' and 'coping' revealed a significant improvement following the smartphone-guided educational treatment with effect sizes of .566 and .285 respectively. No significant improvements were found for the subscales "confidence", "self-efficacy" and "self-awareness". The TES is a new scale that was developed and tested in this study. Validation data and norm tables do not exist yet. Therefore, the results need to be interpreted with caution and more research is needed for the development of the tool. With this caution in mind, it can be summarized that there are hints for improvement of self-rated health literacy and tinnitusrelated coping strategies following the use of smartphone-guided educational treatment. For future studies, we would also suggest the measurement of the empowerment at several time points during the treatment. This could be used to describe the health journey in more detail and investigate if certain aspects of the empowerment are more important at specific time points of the health journey (see e.g. [30]). As an example, with a larger sample it 
would be possible to analyze if there is an influence of the tinnitus duration, the number of comorbidities or traumatic life events.

Dropouts. Between study allocation and final assessment, 26 of 62 participants dropped out of the study. The reasons are listed in figure 1. A closer analysis of the reasons showed that in 16 of 26 the reasons can be addressed by technical improvements. A leading cause for dropout was the installation process of the app via testflight, which was more complicated than the installation of an ordinary app via the appstore. Furthermore, participants dropped out because the app did not run on their smartphone (e.g. low iOS version or Android). Although technical requirements were clearly communicated during the recruitment process, several participants ended up in the study without a suitable smartphone. Future studies can address these technical issues if they provide an app for both systems, iOS and Android, and make the app available via the respective appstores.

\subsection{Conclusion and future directions}

With this feasability study, we explored the feasibility of providing educational training on the smartphone without the guidance of a medical person. We observed small to medium improvements on tinnitus distress measures, but not on tinnitus loudness. Patients with a greater commitment to the intervention showed stronger improvements in tinnitus distress. It can be hypothesized that these improvements are related to the observed improvements in tinnitus-specific health literacy and coping strategies. Technical and methodological improvements for future work in this area is discussed. With the use of modern smartphone technology, there is a huge potential that can be unlocked in future studies: e.g. with more detailed knowledge of the individual patient and knowledge of large-scale crowdsensing patient data, it would be possible to deliver personalized tips and counseling to the patient [31]. Furthermore, with a continuous evaluation of symptoms related to tinnitus, it would be possible to predict the health condition of the individual tinnitus patient [24] and deliver tips and tinnitus-specific knowledge to the patient at times when it is most important. 
Author Contributions: Conceptualization methodology: WS, BL, RP, JS, RH, RP; Investigation and project administration: WS, HS, MN; Writing and revision of the manuscript: WS, PN, JS, BL, SS, MS, JS, RH, RP.

Funding: The research study on and the development of the TinnitusTipps mHealth app was funded by Sivantos $\mathrm{GmbH}$. This work is partially supported by the European Union's Horizon 2020 Research and Innovation Programme, Grant Agreement Number 848261 for UNITI-Unification of Treatments and Interventions for Tinnitus Patients [32].

Institutional Review Board Statement: The study was conducted according to the guidelines of the Declaration of Helsinki, and approved by the Research Ethics Committees of the University Hospital Regensburg (protocol number: 17-544_1-101).

Conflicts of Interest: $\mathrm{RH}$ is/was employed by Sivantos $\mathrm{GmbH}$. The mHealth app and the study received funding from Sivantos $\mathrm{GmbH}$. The funder had the following involvement: conceptualization of the App and the pilot-study, comments and feedback on the manuscript. WS and RP are also stakeholders of the Lenox $\mathrm{uG}$, which aims to translate scientific knowledge into e-health applications. WS is coordinator of EU (Horizon 2020 framework) funded "Unification of Treatments and Interventions for Tinnitus Patients" (UNITI, grant no. 848261). 


\section{References}

1. Møller, A.R. Textbook of Tinnitus. In; Textbook of Tinnitus; 2011; pp. 25-27 ISBN 9781607611448.

2. Ridder, D.D.; Schlee, W.; Vanneste, S.; Londero, A.; Weisz, N.; Kleinjung, T.; Shekhawat, G.S.; Elgoyhen, A.B.; Song, J.-J.; Andersson, G.; et al. Tinnitus and Tinnitus Disorder: Theoretical and Operational Definitions (an International Multidisciplinary Proposal). Progress in brain research 2021.

3. Biswas, R.; Lugo, A.; Akeroyd, M.A.; Schlee, W.; Gallus, S.; Hall, D.A. Tinnitus Prevalence in Europe: A Multi-Country CrossSectional Population Study. Lancet Regional Heal - Europe 2021, 100250, doi:10.1016/j.lanepe.2021.100250.

4. McCormack, A.; Edmondson-Jones, M.; Somerset, S.; Hall, D. A Systematic Review of the Reporting of Tinnitus Prevalence and Severity. Hearing Research 2016, 337, 70-79.

5. Gallus, S.; Lugo, A.; Garavello, W.; Bosetti, C.; Santoro, E.; Colombo, P.; Perin, P.; Vecchia, C.L.; Langguth, B. Prevalence and Determinants of Tinnitus in the Italian Adult Population. Neuroepidemiology 2015, 45, 12-19.

6. Baguley, D.; McFerran, D.; Hall, D. Tinnitus. The Lancet 2013.

7. Gallus, S.; Lugo, A.; Garavello, W.; Bosetti, C.; Santoro, E.; Colombo, P.; Perin, P.; Vecchia, C.L.; Langguth, B. Prevalence and Determinants of Tinnitus in the Italian Adult Population. Neuroepidemiology 2015, 45, 12-19, doi:10.1159/000431376.

8. Landgrebe, M.; Zeman, F.; Koller, M.; Eberl, Y. The Tinnitus Research Initiative (TRI) Database: A New Approach for Delineation of Tinnitus Subtypes and Generation of Predictors for Treatment Outcome. ... and decision making 2010.

9. Elgoyhen, A.B.; Langguth, B.; Ridder, D.D.; Vanneste, S. Tinnitus: Perspectives from Human Neuroimaging. Nature Reviews Neuroscience $2015,16,632-642$.

10. Cederroth, C.R.; Gallus, S.; Hall, D.A.; Kleinjung, T.; Langguth, B.; Maruotti, A.; Meyer, M.; Norena, A.; Probst, T.; Pryss, R.; et al. Editorial: Towards an Understanding of Tinnitus Heterogeneity. Front Aging Neurosci 2019, 11, 53, doi:10.3389/fnagi.2019.00053.

11. McFerran, D.J.; Stockdale, D.; Holme, R.; Large, C.H.; Baguley, D.M. Why Is There No Cure for Tinnitus? Front Neurosci-switz 2019, 13, 802, doi:10.3389/fnins.2019.00802.

12. Fuller, T.; Cima, R.; Langguth, B.; Mazurek, B.; Vlaeyen, J.W.; Hoare, D.J. Cognitive Behavioural Therapy for Tinnitus. Cochrane Db Syst Rev 2020, 2020, CD012614, doi:10.1002/14651858.cd012614.pub2.

13. Schoisswohl, S.; Langguth, B.; Schecklmann, M.; Bernal-Robledano, A.; Boecking, B.; Cederroth, C.R.; Chalanouli, D.; Cima, R.; Denys, S.; Dettling-Papargyris, J.; et al. Unification of Treatments and Interventions for Tinnitus Patients (UNITI): A Study Protocol for a Multi-Center Randomized Clinical Trial. Trials 2021, 22, 875, doi:10.1186/s13063-021-05835-z.

14. Henry, J.A.; Stewart, B.J.; Griest, S.; Kaelin, C.; Zaugg, T.L.; Carlson, K. Multisite Randomized Controlled Trial to Compare Two Methods of Tinnitus Intervention to Two Control Conditions. Ear Hearing 2016, 37, e346-e359, doi:10.1097/aud.0000000000000330. 
15. Xiang, T.; Zhong, J.; Lu, T.; Pu, J.; Liu, L.; Xiao, Y.; Lai, D. Effect of Educational Counseling Alone on People with Tinnitus: Meta-Analysis of Randomized Controlled Trials. Patient Educ Couns 2020, 103, 44-54, doi:10.1016/j.pec.2019.07.031.

16. Tunkel, D.E.; Bauer, C.A.; Sun, G.H.; Rosenfeld, R.M.; Chandrasekhar, S.S.; Cunningham, E.R.; Archer, S.M.; Blakley, B.W.; Carter, J.M.; Granieri, E.C.; et al. Clinical Practice Guideline. Otolaryngology Head Neck Surg 2014, 151, S1-S40, doi:10.1177/0194599814545325.

17. Cima, R.F.F.; Mazurek, B.; Haider, H.; Kikidis, D.; Lapira, A.; Noreña, A.; Hoare, D.J. A Multidisciplinary European Guideline for Tinnitus: Diagnostics, Assessment, and Treatment. Hno 2019, 67, 10-42, doi:10.1007/s00106-019-0633-7.

18. Biesinger; E. Tinnitus - Endlich Ruhe Im Ohr: Ursachen Erkennen Und Ausschalten; Goldmann Taschenbuch Verlag: München, 2012;

19. Hallam; R. Leben Mit Tinnitus: Wie Ohrgeräusche Erträglicher Werden; Quintessenz: Berlin, 1994;

20. Hesse; G. Therapie Bei Chronischem Tinnitus: Habituationstherapien; Thieme, 2016;

21. Kellerhals; Zogg, B.\& Tinnitus-Hilfe: Ein Arbeitsbuch Für Patienten Und Ihre Ärztlichen Und Nichtärztlichen Helfer; Karger, 2004;

22. Ross; U.H. Tinnitus: So Finden Sie Wieder Ruhe; GU-Ratgeber, 2009;

23. Schlee, W.; Pryss, R.C.; Probst, T.; Schobel, J.; Bachmeier, A.; Reichert, M.; Langguth, B. Measuring the Moment-to-Moment Variability of Tinnitus: The TrackYourTinnitus Smart Phone App. Front Aging Neurosci 2016, 8, 294, doi:10.3389/fnagi.2016.00294.

24. Unnikrishnan, V.; Schleicher, M.; Shah, Y.; Jamaludeen, N.; Pryss, R.; Schobel, J.; Kraft, R.; Schlee, W.; Spiliopoulou, M. The Effect of Non-Personalised Tips on the Continued Use of Self-Monitoring MHealth Applications. Brain Sci 2020, 10, 924,

doi:10.3390/brainsci10120924.

25. Newman, C.W.; Jacobson, G.P. Development of the Tinnitus Handicap Inventory; ... -Head \& Neck ...; 1996;

26. Genitsaridi, E.; Partyka, M.; Gallus, S.; Lopez-Escamez, J.A.; Schecklmann, M.; Mielczarek, M.; Trpchevska, N.; Santacruz, J.L.; Schoisswohl, S.; Riha, C.; et al. Standardised Profiling for Tinnitus Research: The European School for Interdisciplinary Tinnitus Research Screening Questionnaire (ESIT-SQ). Hearing Res 2019, 377, 353-359, doi:10.1016/j.heares.2019.02.017.

27. Gastal, D.A.; Pinheiro, R.T.; Vazquez, D.P. Self-Efficacy Scale for Brazilians with Type 1 Diabetes. Sao Paulo Med J 2007, 125, 96101, doi:10.1590/s1516-31802007000200006.

28. Pagliarello, C.; Di Pietro, C.; Paradisi, A.; Abeni, D.; Tabolli, S. Measuring Empowerment in Patients with Psoriasis: The Psoriasis Empowerment Enquiry in the Routine Practice (PEER) Questionnaire. Eur J Dermatol 2010, 20, 200-204, doi:10.1684/ejd.2010.0893.

29. Team, R.C. R: A Language and Environment for Statistical Computing. 2019, 1-16.

30. Gotowiec, S.; Larsson, J.; Incerti, P.; Young, T.; Smeds, K.; Wolters, F.; Herrlin, P.; Ferguson, M. Understanding Patient Empowerment along the Hearing Health Journey. Int J Audiol 2021, 1-11, doi:10.1080/14992027.2021.1915509. 
31. Simoes, J.; Neff, P.; Schoisswohl, S.; Bulla, J.; Schecklmann, M.; Harrison, S.; Vesala, M.; Langguth, B.; Schlee, W. Toward Personalized Tinnitus Treatment: An Exploratory Study Based on Internet Crowdsensing. Frontiers Public Heal 2019, 7, 157, doi:10.3389/fpubh.2019.00157.

32. Schlee, W.; Schoisswohl, S.; Staudinger, S.; Schiller, A.; Astrid, L.; Berthold, L.; Martin, S.; Jorge, S.; Patrick, N.; Dimitris, K. Towards a Unification of Treatments and Interventions for Tinnitus Patients: The EU Research and Innovation Action UNITI. Progress in brain research. 